\title{
Recherche en soins palliatifs: la Suisse rattrape son retard
}

\author{
Myriam Tapernouxa , Sarah Fasolin ${ }^{b}$
}

${ }^{a}$ responsable du ressort Science de I'ASSM; b journaliste indépendante

De nombreuses personnes ont besoin d'un traitement palliatif dans la dernière phase de leur vie. Mais précisément dans ce domaine, si important pour la médecine et la société, la Suisse était encore à la traîne par rapport à d'autres pays, y compris en matière de recherche. En 2014, l’Académie Suisse des Sciences Médicales (ASSM) a lancé le programme de soutien «Recherche en soins palliatifs 2014-2018» doté d'un montant total de 4,4 millions de francs. Une publication en présente les résultats.

Comme le rappelle la professeure genevoise Sophie Pautex, co-fondatrice de la "plateforme nationale de recherche en soins palliatifs» et membre de la commission d'évaluation du programme de soutien de l'ASSM, on ne comptait en 2008 que 12 articles sur le thème des soins palliatifs publiés par des équipes de recherche suisses. Un chiffre modeste par rapport à l'importance des soins palliatifs dans le quotidien médical. En effet, en Suisse, près de $90 \%$ de la population décède aujourd'hui non pas de mort subite, mais après une période de maladie et de soins. La fin de vie est ainsi, pour la plupart des personnes, un processus de durée variable.

Il y a une dizaine d'années, ce n'était pas uniquement les activités de recherche qui étaient encore insuffi-

\footnotetext{
L'essentiel en bref

Les soins palliatifs sont omniprésents dans le quotidien médical suisse et prennent de plus en plus d'importance en raison de l'évolution démographique.

Le programme «Recherche en soins palliatifs 2014-2018» de I'ASSM, financé par la Fondation StanleyThomas Johnson et la Fondation Gottfried et Julia Bangerter-Rhyner, a permis de soutenir 34 projets et d'octroyer quatre bourses.

Ce programme s'est inscrit dans le cadre d'une offensive de recherche lancée par la Confédération et les cantons pour rattraper le retard de la Suisse par rapport à d'autres pays dans le domaine des soins palliatifs.

Le symposium de clôture organisé par I'ASSM, auquel ont participé 120 professionnels des soins palliatifs, a montré que de nombreux progrès avaient pu être réalisés mais qu'une poursuite du soutien ainsi que des financements additionnels étaient nécessaires.
}

santes, mais aussi l'éventail des services offerts aux patientes et aux patients. La Confédération et les cantons ont alors lancé la «Stratégie nationale en matière de soins palliatifs 2010-2015", dans le but d'encourager tous les domaines des soins palliatifs: la sensibilisa-

Parmi les 138 requêtes examinées, 34 projets et quatre bourses d'un montant total de 4,4 millions de francs ont été financés.

tion, les soins, la formation et le financement et la recherche. Dans ce contexte, le Conseil fédéral a lancé le «Programme national de recherche Fin de vie» (PNR 67) et l'ASSM, quant à elle, le programme de soutien «Recherche en soins palliatifs 2014-2018».

Dans le cadre de ce programme, un total de 138 requêtes a été examiné; 34 subsides et 4 bourses pour des séjours de formation et de recherche à l'étranger ont été financés. Le domaine de l'oncologie a été délibérément exclu, car la recherche dans ce domaine bénéficie déjà d'autres sources de financement. Les projets portaient sur le traitement des symptômes physiques (comment les douleurs peuvent-elles être soulagées?), sur l'accompagnement psychologique et spirituel (comment peut-on, par exemple, répondre à des questions sur le sens de la vie? Comment les proches sont-ils impliqués?), sur des thèmes en lien avec l'éthique (quelles sont les décisions envisageables - par exemple, mort volontaire - et souhaitées par le patient?), et également sur des problématiques liées à l'infrastructure et aux procédures (le patient doit-il être pris en charge à son domicile ou en milieu hospitalier?). 
Pour répondre à toutes ces questions, l'ASSM a pu compter sur l'engagement de deux fondations privées: la Fondation Stanley Thomas Johnson et la Fondation Gottfried et Julia Bangerter-Rhyner qui ont soutenu le programme avec un montant total de 4,4 millions de francs.

\section{Des données plus sûres, des réseaux plus solides}

Il n'est pas possible de dresser un bilan définitif, car certains projets sont encore en cours. Toutefois, on peut d'ores et déjà affirmer que le programme a considérablement amélioré la situation des données dans le domaine des soins palliatifs. Par ailleurs, des réseaux de recherche ont été créés, l'échange entre les hôpitaux et les autres institutions s'est intensifié et de nombreuses publications sont parues. Il convient également de noter les progrès réalisés dans le domaine

\section{L'évolution démographique le montre: le vieillissement croissant de la population rend les soins palliatifs d'autant plus importants.}

de la relève: des jeunes chercheuses et chercheurs ont bénéficié du programme en collaborant à des projets ou en étant directement soutenus avec une bourse.
Le programme de soutien a été officiellement conclu avec le symposium «Research in Palliative Care» qui s'est déroulé à Berne, le 21 novembre 2019. Environ 120 professionnels, actifs au quotidien dans les soins palliatifs, se sont penchés sur les questions que se pose la recherche sur la fin de vie. Les résultats du programme ont été présentés et les défis auxquels sont confrontés les chercheurs ont été discutés. L'importance de poursuivre la recherche en soins palliatifs en Suisse a été soulignée à maintes reprises lors de cette journée.

Pour être attractive pour la relève, la recherche en soins palliatifs devrait être mieux ancrée dans les institutions académiques.

Un regard vers l'avenir suffit pour s'en convaincre: tout comme l'âge moyen de la population, l'importance des soins palliatifs va elle aussi augmenter. Selon l'Office fédéral de la statistique, la proportion de la population de plus de 60 ans en Suisse s'élève aujourd'hui à $25,4 \%$. En 2050, elle sera de 33,8\%.

a publication «Recherche en soins palliatifs. Rapport final sur le programme de soutien 2014-2018 de I'ASSM» est disponible gratuitement sous forme imprimée ou électronique: assm.ch soins-palliatifs

Aperçu de quelques projets

Dans le domaine des soins palliatifs, divers groupes professionnels travaillent en étroite collaboration. L'entourage de la personne concernée est également sollicité pour qu'elle puisse bénéficier de la meilleure qualité de vie possible jusqu'à sa mort. L'étendue du sujet se reflète également dans la variété des questions de recherche examinées dans le cadre du programme de soutien de l'ASSM.

\section{A la recherche de données fiables}

Quels patients ont accès aux soins palliatifs en Suisse? Comment se déroule leur vie dans ces institutions? Pour étudier ces questions, une équipe de recherche dirigée par Steffen Eychmüller de l'Inselspital à Berne et Sophie Pautex des HUG a adressé des questionnaires à six services de soins palliatifs en Suisse. 379 questionnaires ont été évalués: en moyenne, une personne traitée dans un service de soins palliatifs est âgée de 73 ans, est une femme, de confession protestante et est atteinte d'un cancer. L'étude a montré que les patients ne souffrant pas d'un cancer accédaient plus difficilement aux soins palliatifs. Les chercheurs souhaitaient également démontrer qu'il serait possible de constituer une base de données nationale pour les soins palliatifs. Le projet devrait être poursuivi dans le but d'institutionnaliser un outil de collecte des données correspondant en Suisse.

\section{Lorsque des personnes cessent de s'alimenter et de s'hydrater}

La plupart des patients avaient environ 80 ans et souffraient d'une maladie mortelle. Leur espérance de vie était in férieure à une année. Parce qu'ils avaient peur de devenir dépendants et étaient épuisés, ils ont décidé d'arrêter de 
s'alimenter et de s'hydrater. Dans la plupart des cas, les personnes concernées sont décédées après environ deux semaines. Tels sont les résultats d'une première enquête nationale concernant le Renoncement Volontaire à l'Alimentation et à l'Hydratation (RVAH) menée par une équipe de recherche dirigée par André Fringer de la Zürcher Hochschule für Angewandte Wissenschaften (ZHAW) dans le cadre d'une étude empirique. Certes, il s'agit, dans le quotidien médical, d'un phénomène marginal - seuls 1,7\% des patientes et des patients qui décèdent dans les institutions de soins choisissent cette voie. Pour les professionnels concernés, le RVAH peut néanmoins représenter un défi majeur et les confronter à un dilemme éthique. Selon les chercheurs, les résultats de l'étude et les connaissances scientifiques actuelles ont démontré qu'un débat professionnel, par exemple au moyen de mesures de formation sur le RVAH, était nécessaire.

\section{Soins palliatifs à domicile pour les patients SLA}

La sclérose latérale amyotrophique (SLA) est une maladie dégénérative du système nerveux pour laquelle il n'existe pas de traitement. Du diagnostic à la mort, la durée de vie des patients souffrant de SLA est généralement de deux à trois ans. Une période pendant laquelle ces derniers sont souvent pris en charge à leur domicile par un ou plusieurs proches jusqu'à leur décès. Une équipe de recherche dirigée par Tenzin Wangmo de l'Institut d'éthique biomédicale de l'Université de Bâle a étudié les besoins des proches aidants en termes de soutien, tant du point de vue émotionnel que physique, pendant cette période difficile.

\section{De la gratitude pour une vie qui touche à sa fin}

Celles et ceux qui peuvent témoigner de la gratitude à leurs proches traversent la dernière phase de leur vie avec plus de sérénité et de solidité. C'est du moins ce que pensent les chercheuses et les chercheurs de l'équipe de Mathieu Bernard du CHUV. Car, selon des projets de recherche antérieurs, les facteurs non physiques peuvent avoir une influence considérable sur la qualité de vie d'un patient et de sa famille. Mais comment devient-on reconnaissant? Et comment les malades peuvent-ils être soutenus? Pour leur étude, les chercheurs ont procédé à des interventions de gratitude avec 30 binômes de patients-proches. Celles-ci se déroulent en deux étapes. La première consiste en la rédaction d'une lettre de gratitude, dans laquelle le patient et les proches écrivent pourquoi ils sont reconnaissants envers l'autre. La deuxième étape est une visite de gratitude, au cours de laquelle les lettres sont échangées et lues à haute voix ou remises pour une lecture ultérieure.

\section{Opération cardiaque et/ou soins palliatifs?}

Le taux de mortalité des patients âgés présentant une sténose aortique symptomatique est très élevé. Une équipe de recherche dirigée par Tanja Krones, de l'Hôpital universitaire de Zurich, tente de découvrir par le biais d'entretiens quels sont les souhaits de ces patients quant à la planification actuelle de leur traitement et en cas de futures crises. Le patient souhaite-t-il se soumettre à une opération à cœur ouvert ou à une intervention cardiaque moins invasive? A quel moment privilégie-t-il un plan de traitement plus orienté vers les soins palliatifs? Et tout particulièrement, comment agir en cas de complications pendant ou après l'opération? L'analyse des entretiens servira de base à l'élaboration d'outils décisionnels. Cet instrument devrait permettre au patient, aux proches et aux équipes de soins interprofessionnelles de trouver plus facilement une voie harmonieuse entre les possibilités médicales, les chances et les complications attendues.

\section{La mort en début de vie}

Chaque année, environ 600 bébés décèdent en Suisse entre la $22^{\mathrm{e}}$ semaine de grossesse et la première semaine après la naissance. Ces cas de décès périnatals sont, pour les parents concernés, une épreuve particulièrement douloureuse qui peut, dans les cas les plus sévères, provoquer des troubles de stress post-traumatique, des dépressions et d'autres problèmes encore. Une équipe de recherche, dirigée par Claudia Meier Magistretti de la Haute école de Lucerne et Valerie Fleming de l'University Liverpool, a déterminé, par le biais d'entretiens avec des parents concernés, quelle prise en charge et quels soutiens peuvent être envisagés pour éviter des conséquences psychiques à long terme. Un modèle de bonne pratique développé en collaboration avec des parents, des experts et des assurances maladie définit diverses mesures destinées à soutenir efficacement les parents concernés depuis le moment du diagnostic jusqu'au travail de deuil. 